\title{
Tangence
}

\section{Remarques au profit des jeunes littérateurs}

\section{François Tétreau}

Numéro 53, décembre 1996

L’humour de la poésie

URI : https://id.erudit.org/iderudit/025923ar

DOI : https://doi.org/10.7202/025923ar

Aller au sommaire du numéro

Éditeur(s)

Tangence

ISSN

0226-9554 (imprimé)

1710-0305 (numérique)

Découvrir la revue

Citer cet article

Tétreau, F. (1996). Remarques au profit des jeunes littérateurs. Tangence, (53), 8-12. https://doi.org/10.7202/025923ar d'utilisation que vous pouvez consulter en ligne.

https://apropos.erudit.org/fr/usagers/politique-dutilisation/ 


\section{Remarques au profit des jeunes littérateurs François Tétreau}

\section{Nature de l'humour}

Je ne vois pas que l'humour en poésie soit bien différent de l'humour en prose, sinon qu'il tient à des mots plutôt qu'à des tournures. Encore que. Mais ne disputons pas trop là-dessus. Ce qui va pour l'une ira pour l'autre ici, et voilà pourquoi les lumineuses remarques subséquentes seront d'ordre général.

En premier lieu, il convient de savoir de quel humour on parle. Vrai. Si le lecteur ne se doute pas que tel auteur - prosateur ou poète -, joue sur le mode ironique, s'il ne lui prête aucun recul, s'il ne lui reconnaît pas d'emblée une quelconque position critique, par quelle magie, dites-moi, saisira-t-il son humour. Les germanophones tiennent Kafka pour un humoriste et les Américains affirment la même chose à propos de Faulkner. Quelle différence entre une observation du Dictionnaire des idées reçues et celle de Bernardin de Saint-Pierre, selon laquelle le melon est divisé par la nature pour être mangé en famille. On s'aviserait de lire Flaubert au pied de la lettre, qu'on n'entendrait pas grand chose à ses sarcasmes. Qui sait si Bernardin de SaintPierre ne fut jamais pince-sans-rire.

Une foule de gens graves ouvrent en littérature, qui écrivent lourdement, et sont risibles à leur insu. Cela pimente le jeu, mais cela le gauchit également, car l'humour, enfin, n'est pas tant le comique. De là l'importance de poser d'entrée de jeu un premier axiome: l'humour procède d'une intention.

\section{Identité du cuistre}

Bien. Maintenant, de quelle intention s'agit-il. Hum. Nous pourrions spéculer longtemps, mais allons-y hardiment d'une hypothèse: l'humour procède de l'intention de faire rire aux dépens d'un tiers.

Reste à identifier ce tiers. Travail d'autant plus périlleux que le tiers et le quart n'ont de cesse de se métamorphoser au fil des ans. Un exemple. On imagine mal aujourd'hui un poète québé- 
cois de vingt ans se moquant des curés (ce serait plutôt l'inverse). Que dire alors d'un poème d'autrefois prenant à partie les curés sur le mode plaisant. Sentira-t-on son esprit? En conscience, nous ne nous en amuserions guère, si nous ne pouvions compter sur la formidable capacité des tierces personnes à se reproduire continûment. Examinons la chose suivante. Il y a, il y eut, il y aura toujours, une nuée de bien-pensants résolus à nous sermonner. C'est fort agaçant, mais ces personnes, par bonheur, se démasquent facilement. Supposons que les curés aient été les bienpensants d'une certaine époque et qu'un poète plus ou moins provincial les ait alors épinglés dans un recueil. Il nous suffira de substituer, durant notre lecture, les bien-pensants actuels à ces curés anciens, pour retrouver intacte l'initiale ironie du poète. Ainsi les grands écrivains font d'une pierre bien des coups. Dans la meilleure des musiques, ils moquent les emmerdeurs de leur temps, au grand soulagement de leurs contemporains, et nous fournissent d'excellents motifs pour que nous puissions à notre tour chanter les ridicules des emmerdeurs nouveau genre. Sans doute chacun d'entre nous a-t-il son souffre-douleur intime, qu'il tourmente de ses tracasseries particulières. Ce sera tantôt un médecin, un directeur, un patron, ou un propriétaire, mais de nos jours, je n'invente rien, c'est écrit en première page du Monde diplomatique et cela fait l'objet d'ouvrages extrêmement sérieux publiés aux USA, ceux qui infligent leurs valeurs, leur morale, leur manière de voir à toute la tribu, et qui rabrouent les naïfs osant mettre en doute la pureté de leurs intentions, sont bien évidemment ceux-ci qui "se prostituent dans les pissotières du journalisme». Comme nous le signale Léon Bloy. Ce n'est qu'un mauvais moment à passer, d'autres prendront un jour la place, mais à l'heure actuelle, les gazetiers répondent trait pour trait au signalement, que nous a courtoisement transmis Molière, des emmerdeurs haut de gamme. Les marquis de La Jambe officient à la télévision et les sujets de ridicule abondent.

\section{D'une académie de mot}

De nombreux esprits chagrins, forts en gueule de surcroît, décèlent du maniérisme où il n'est que bonne humeur. L'affaire se complique de ceci que certaines gens, dont le métier est de goûter la fantaisie, sont dans l'ensemble ces esprits chagrins. Enfin l'histoire s'embrouille tout à fait quand ces personnes, si promptes à débusquer l'effet précieux et à tonner là contre, écri- 
vent elles-mêmes de la façon la plus maniérée qui soit. Un tel se gargarise de subjonctifs itératifs qu'il ne maîtrise même pas, et tel autre (ou le même) se prend à chapitrer des auteurs aux blanches mains et à leur reprocher un galimatias pourtant moins obscur que le sien. On comprendra qu'un poète de dix-neuf ans ne s'y retrouve guère et qu'il y a nécessité de prodiguer de nouveaux conseils à l'endroit des jeunes littérateurs.

Avant le sens, il existe la forme. Telle femme, avant d'être Isabelle ou Sophie, est une femme. Rien de drôle là-dedans, mais il importe de noter que la physionomie précède le sens. Il en va de même pour les vocables. En poésie, l'emploi d'un mot curieux, singulier d'aspect, ou inattendu, par un sentencieux, ne présage rien de très amusant, alors que le même mot dans un texte de Fargue prend immédiatement un tout autre relief. La préciosité reste le fait du précieux, elle ne réside pas dans les mots, qui appartiennent à tout le monde. S'il en était autrement, on éliminerait du dictionnaire tous les mots dits précieux et il n'y aurait plus de préciosité. On voit que cela n'a pas de sens. Les mots possèdent une anatomie. Vous, par exemple, qui ne savez pas l'italien, vous repérez sans peine, dans un texte écrit en italien, des mots ayant une physionomie plus amusante que les autres. Il découle de cela que tel vocable bien amené chatouillera vos lectrices à l'envi.

D'où le deuxième axiome, non moins capital que le premier: les hommes ne savent pas, mais les jeunes lectrices comprennent.

\section{D'une stratégie de l'encerclement}

Pour y voir mieux, je recommande à chacun de se pénétrer de la vérité suivante. Les gazetiers, c'est dans leur nature, morigènent. On aura beau soupirer, nous n'y ferons rien. Cela étant noté, il existe, c'est malheureux à dire mais il existe effectivement, des individus primaires exposant d'épaisses idées. Ce qu'un lecteur sain d'esprit détecte sans faillir. En deuxième ligne, se dressent des sergents de presse, qui passent leur temps à vilipender les primaires et qui en voient partout. On dira que ce travail est d'hygiène publique, il n'empêche. À force de sévir durant des années derrière le même pupitre, ces représentants de l'ordre littéraire réussissent à transformer les épaisses idées en autant d'interdits, dont ils assomment les braves gens. Survient un jour un jeune facétieux qui, fatigué par tant de remontrances, expose des 
idées, d'apparence épaisse, avec l'intention bien légitime de faire suer les gendarmes de la chose écrite. Alors se produit un phénomène, et loufoque et jouissif, il arrive ceci que les gazetiers n'entendent absolument pas l'ironie du facétieux, pour cette raison qu'il ne leur vient pas un instant à l'esprit qu'on puisse l'exercer à leurs dépens. Étant entendu que le rendement d'une blague est décuplé par l'incompréhension d'une tierce personne, et que les tierces personnes sont légion, on mesure l'euphorie qui nous est promise.

\section{Du pètesec}

Une mise en garde cependant. Les bien-pensants d'aujourd'hui ayant combattu leurs prédécesseurs sur ce point, le sexe n'est plus tabou. Encore moins la grossièreté. Évolution que les échotiers considèrent comme étant leur propre victoire, après des décennies de valeureux services au marbre. Enfin il n'y a plus de marbre nulle part mais ces gens s'expriment ainsi. Là-dessus, intervention d'un poète de dix-huit ans : quelle évolution y a-t-il à chasser un tabou, si ceux-là qui consacrent leur vie à son éradication, le remplacent illico par un autre. En vérité, mon ami, je vous le dis, vous touchez là au vif du sujet et il s'agit de ne pas se méprendre, car combien d'écrivains actuels portent l'érotisme jusqu'à des outrances à peine imaginables, dans l'espoir uniquement qu'un journaliste les rappelle à l'ordre et, partant, se trahisse. Voyons. Mauvaise, très mauvaise politique. On se trompe de terrain. Il n'y a plus d'ennemis sur ce front-là. Pour l'instant du moins. En procédant de la sorte, ces écrivains consciencieux, mais distraits, offrent aux échotiers l'occasion d'étaler leur tolérance, leur immense ouverture d'esprit, et cela sur un ton de condescendance à peine supportable. Non et non. Ces auteurs ratent tout à fait leur cible en comblant l'adversaire. Ce n'est pas la bonne méthode. En revanche si un jeune homme aux cheveux verts, non moins rusé que le facétieux de tout à l'heure, s'autorisait à donner un récit narquois à propos, mettons, des ethnies, pas un seul gazetier, je vous le donne en mille, ne décèlerait l'intention d'abord, c'est-à-dire la moquerie dirigée, non pas contre les ethnies bien sûr, mais contre les fâcheux, et toute la caste ensuite condamnerait le brûlot sans appel. A-t-on le droit d'aborder ainsi semblable sujet. Les bien-pensants sont aussi chatouilleux sur certaines questions que l'étaient jadis les curés sur celle du sexe. Comme les pauvres gens qui s'entourent de 
bibelots, les bien-pensants ne souffrent pas de vides dans le code. Il leur faut du tabou. On s'en remettra aux pathologistes pour y comprendre quelque chose, mais les faits sont ainsi. Il y a chez eux un besoin morbide de jouer de la férule et d'imposer leurs déplaisirs. Qu'à cela ne tienne. Les jeunes littérateurs ont mieux à faire, et vient ici le moment, pour eux, de ruminer un troisième axiome sous forme de conseil: quand tout le monde écrit comme une marquise, retrouve la langue du paysan et, à l'inverse, lorsque les contempteurs jouent les pétomanes, sors ton éventail.

\section{Ultimes considérations}

Que ce soit en prose, ou en poésie, ce n'est pas moi qui le dis, on dénombre trois catégories d'individus. Il y ceux qui emploient l'ironie, ceux qui la goûtent, et ceux à qui elle échappe. À la vérité, on ne sait rien de plus moderne que ça. Dans un poème, tel mot tombe à point, qui échappe aux tierces personnes, mais qui agit tout à la fois, comme agit la fumée du cigare sur l'odorat des censeurs. Il vaut toujours mieux avoir les fumeurs de son côté.

\section{$* * *$}

Il est rare qu'on développe une plaisanterie sur tout un poème. À moins d'être Villon. "Par ce testament, je lègue à mon créancier mes chaussettes, dont j'ai souvent tiré les élastiques, aux gendarmes qui m'ont ramené dans la bonne voie, je lègue cette liasse de poèmes que m'avait confiée mon ami, et à mon courriériste privilégié, je laisse Lise et Charlotte, mes oies, qui tant m'ont appris, etc., etc. " 